\title{
Desenvolvimento de Método Alternativo para o Ensino da Técnica do Caminhamento Elétrico
}

Luan Viana dos Santos, Universidade Federal dos Vales do Jequitinhonha e Mucuri

Jackeline Cabral, Universidade Federal dos Vales do Jequitinhonha e Mucuri

Élida Corrêa, Universidade Federal dos Vales do Jequitinhonha e Mucuri

Carlos Henrique Alexandrino, Universidade Federal dos Vales do Jequitinhonha e Mucuri

Copyright 2016, SBGf - Sociedade Brasileira de Geofísica

Este texto foi preparado para a apresentação no VII Simpósio Brasileiro de Geofísica Ouro Preto, 25 a 27 de outubro de 2016. Seu conteúdo foi revisado pelo Comitê Técnico do VII SimBGf mas não necessariamente representa a opinião da SBGf ou de seus associados É proibida a reprodução total ou parcial deste material para propósitos comerciais sem prévia autorização da SBGt.

\section{Resumo}

A utilização de métodos geofísicos vem crescendo nos últimos anos, com aplicações nas mais diversas áreas, como prospecção de água e identificação de minérios. 0 ensino das técnicas necessárias se dá de forma prática, através da realização de atividades de campo. Algumas universidades, no entanto, não dispõem de condições adequadas para a realização destas atividades. Este projeto, desenvolvido por estudantes de Engenharia Hídrica, elaborou um sistema alternativo e eficaz para possibilitar práticas de métodos geofísicos, especialmente 0 caminhamento elétrico, em universidades com menos recursos, caso da Universidade Federal dos Vales do Jequitinhonha e Mucuri, onde este estudo foi realizado.

\section{Introdução}

Geofísica, segundo a Sociedade Brasileira de Geofísica (SBGf), é o estudo do interior da Terra através de procedimentos realizados na sua superfície. Diferente da geologia, onde os estudos das rochas são feitos através da coleta e análise de amostras retiradas de forma intrusiva, os métodos geofísicos investigam a subsuperfície através dos princípios da Física.

Estudos de terremotos, tsunamis, movimentação das placas tectônicas, prospecção de água e minerais, problemas de engenharia e mapeamento geológico são algumas das aplicações da geofísica (Universidade Federal do Pará - UFPA). A importância dessa ciência e a sua variada gama de aplicações faz com que 0 conhecimento dos métodos geofísicos seja de extrema importância para diversos profissionais, destacando-se, além dos geofísicos, geólogos, arqueólogos e engenheiros.

Por este motivo, inúmeras universidades incluem em suas grades disciplinas que visam ensinar princípios da geofísica bem como alguns dos métodos e técnicas mais populares nesta ciência. Infelizmente, em muitos dos casos, o ensino se restringe a conceitos teóricos devido à dificuldade do ensino dos métodos em campo, por questões logísticas, econômicas, climatológicas e até mesmo didáticas, falta de equipamentos adequados e disponibilidade de cronograma.
Como qualquer outro procedimento prático, o entendimento do funcionamento dos métodos geofísicos se torna menos eficiente quando não se praticam as técnicas estudadas. A busca por alternativas de ensino se tornam então essenciais para estudantes e universidades.

Sabendo-se da dificuldade existente para a prática dos métodos geofísicos em campo, tomando por base a realidade vivida na Universidade Federal dos Vales do Jequitinhonha e Mucuri (UFVJM), os estudantes da disciplina Métodos Geofísicos para Prospecção de Águas Subterrâneas (EHD121), sob orientação do professor doutor Carlos Henrique Alexandrino, desenvolveram um sistema simples e de fácil replicação que possibilita o ensino da geofísica, em especial a técnica do caminhamento elétrico $(\mathrm{CE})$, para alunos dos próximos semestres e de outras universidades que enfrentarem situações semelhantes.

\section{Metodologia}

Visando aperfeiçoar o ensino de métodos geofísicos, especificamente da técnica do caminhamento elétrico (CE), em escolas e universidades, desenvolveu-se um sistema simples e eficaz que pode ser utilizado para realização dos procedimentos exigidos sem a necessidade de visitas e trabalhos de campo.

As relações entre corrente elétrica, potencial elétrico e disposição geométrica dos eletrodos no terreno, permitem calcular a resistividade real ou aparente em subsuperfície.

Os fatores que mais afetam a resistividade dos solos são: composição mineralógica; porosidade; teor em água; quantidade e natureza dos sais dissolvidos.

O trabalho consistiu na utilização do método da eletrorresistividade em uma caixa contendo camadas de diferentes materiais como camadas de brita, sal e solo e um fragmento de rocha.

O solo foi retirado pelos alunos no Campus com a ajuda do discente que adicionou o restante dos materiais. Sobre a caixa foram colocadas linhas como formas de dividi-la em medições (exemplificada na figura 1).

Foram inseridas oito linhas verticais e quatro linhas horizontais. Foram determinadas três profundidades para a realização dos cálculos. São inseridos dois eletrodos, nos primeiros pontos de encontro das linhas horizontais e verticais, que são fixos e tem como função medir a corrente. 
O sistema consiste em uma caixa plástica resistente com dimensões aproximadas $52 \times 41 \times 20$ (largura $\times$ comprimento $x$ altura, em $\mathrm{cm}$ ), onde foram depositados diferentes tipos de materiais com o intuito de simular as diversas camadas do solo. Areia, brita, água, terra e uma pedra de dimensão considerável foram colocados de forma organizada de modo a formar camadas que pudessem ser identificadas através da técnica do CE.

Os procedimentos necessários para a realização do CE foram ensinados previamente aos estudantes através de aulas teóricas e utilização de simuladores livres para melhor compreensão dos processos. Foram fornecidos os materiais necessários, sendo eles um gerador de tensão, um amperímetro e um voltímetro, então, realizaram-se os procedimentos padrões do CE, buscando mensurar a resistividade aparente no maior número de pontos possíveis, para maior eficiência dos resultados.

Com os valores obtidos, realizaram-se as manipulações matemáticas necessárias, feitas através do software LibreOffice e uma planilha com os valores da resistividade aparente em diversos pontos foi elaborada. A planilha foi trabalhada posteriormente no software livre Surfer, oitava versão, a fim de gerar os mapas de arranjo de campo onde podem ser observados a resistividade aparente de cada área do sistema.

Através da interpretação dos mapas, pode-se observar e identificar locais onde a resistividade aparente destoa do esperado, quando comparado as demais camadas, indicando locais onde podem ser encontrados materiais específicos, como poços de água ou minas de minérios.

Os estudantes, devidamente matriculados na disciplina citada previamente, montaram uma simulação da subsuperfície do solo, dentro de uma caixa de plástico resistente e escuro, de modo que as camadas internas não pudessem ser vistas.

O objetivo foi conseguir identificar a existência das diferentes camadas de maneira não-intrusiva, através da técnica do $\mathrm{CE}$, que fora previamente ensinada aos alunos. Foram utilizados uma fonte, um amperímetro e um voltímetro, de pequenas dimensões e capacidades, que não seriam eficientes usados em escala real, sendo, no entanto, os únicos equipamentos disponíveis na UFVJM. Desta forma, os alunos poderiam praticar a técnica sem a necessidade de deslocamento para áreas abertas e utilizando os aparelhos já existentes.

Para a execução da técnica do caminhamento elétrico, a qual visa identificar as camadas do solo horizontalmente, e em diferentes profundidades, fez-se a divisão da caixa em linhas e colunas, para melhor identificação dos pontos onde seriam colocados os dipolos para fornecimento da tensão e medição da corrente e tensão nos diferentes pontos da caixa. A divisão pode ser vista na Figura 1.

Utilizando os aparelhos citados anteriormente, mede-se a corrente e a voltagem em diferentes pontos. Para a obtenção da resistividade aparente, objeto de estudo deste trabalho, fez-se necessário a manipulação dos resultados através de expressões matemáticas, contando com o auxílio do software LibreOffice.

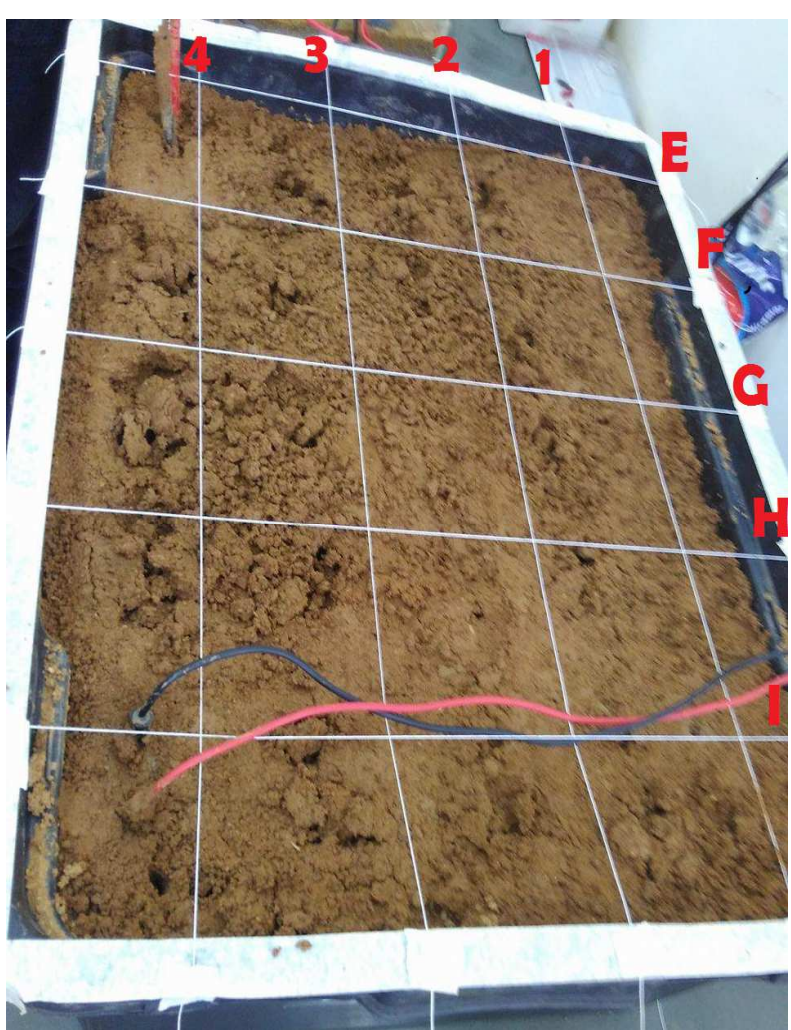

Figura 1 - Divisão das linhas e colunas

$\mathrm{Na}$ Figura 2, vemos uma representação do funcionamento do $C E$, onde os dipolos $A B$ são responsáveis por medir a tensão existente no ponto e os dipolos $\mathrm{MN}$ identificam as correntes. As distâncias entre $\mathrm{AB}, \mathrm{BM}$ e $\mathrm{BN}$ são responsáveis pela variação do ponto e profundidades investigadas. No estudo aqui apresentado, o caminhamento foi executado em dois sentidos, para esquerda e para a direita, a fim de obter maior número de dados.

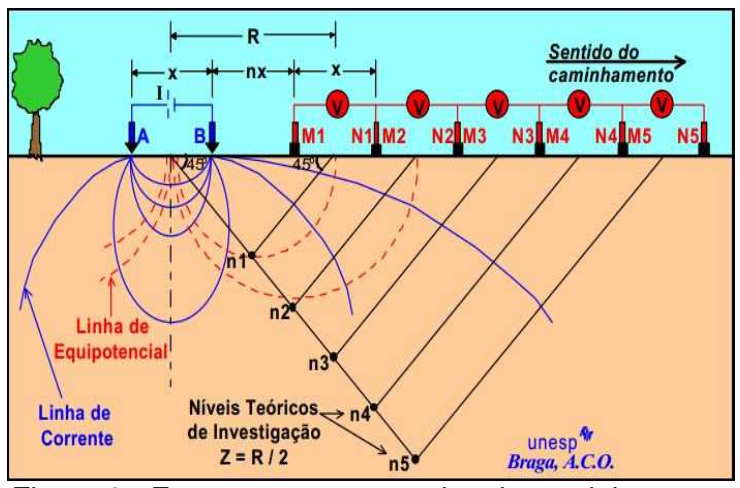

Figura 2 - Esquema representativo do caminhamento elétrico. Fonte: Braga - 2007

Para cálculo da resistividade aparente $\left(\rho_{a}\right)$, utilizou-se a seguinte expressão:

$$
\rho_{a}=K \frac{V}{I}
$$


$\mathrm{Na}$ equação (1) $\mathrm{K}$ e o fator geométrico, $\mathrm{V}$ a diferença do potencial elétrico e I a corrente elétrica. O fator geométrico K é estimado pela equação (2) que depende da disposição dos eletrodos ABMN; $\mathrm{x}=$ espaçamento dos dipolos e $\mathrm{n}=$ nível de investigação correspondente, conforme descrito da equação (3). (BRAGA, 2007).

$$
K=2 \cdot \pi \cdot G \cdot x
$$

e

$$
G=\frac{1}{\frac{1}{n}-\frac{2}{n+1}+\frac{1}{n+2}}
$$

\begin{tabular}{|c|c|c|c|c|c|c|c|c|}
\hline Linha & Sentido $\rightarrow$ & G & v & 1 & K & $\mathrm{Pa}$ & $x$ & P \\
\hline 1 & $\mathrm{~N} 1-\mathrm{AM}=12$ & 3 & 440 & 19,9 & 113,09 & 2500,48 & $6 \mathrm{~cm}$ & $6 \mathrm{~cm}$ \\
\hline 1 & $\mathrm{~N} 2-\mathrm{AM}=24$ & 12 & 890 & 19,94 & 452,38 & 20191,48 & $6 \mathrm{~cm}$ & $12 \mathrm{~cm}$ \\
\hline 1 & $\mathrm{~N} 3-\mathrm{AM}=36$ & 30 & 1801 & 19,3 & 1130,97 & 105537,7 & $6 \mathrm{~cm}$ & $18 \mathrm{~cm}$ \\
\hline 2 & $\mathrm{~N} 1-\mathrm{AM}=12$ & 3 & 391 & 40,1 & 113,09 & 1102,69 & $6 \mathrm{~cm}$ & $6 \mathrm{~cm}$ \\
\hline 2 & $\mathrm{~N} 2-\mathrm{AM}=24$ & 12 & 710 & 39,5 & 452,38 & 8131,38 & $6 \mathrm{~cm}$ & $12 \mathrm{~cm}$ \\
\hline 2 & $2 \mathrm{~N} 3-\mathrm{AM}=36$ & 30 & 323 & 37,5 & 1130,97 & 9741,42 & $6 \mathrm{~cm}$ & $18 \mathrm{~cm}$ \\
\hline 3 & $\mathrm{~N} 1-\mathrm{AM}=12$ & 3 & 378 & 170,5 & 113,09 & 250,72 & $26 \mathrm{~cm}$ & $6 \mathrm{~cm}$ \\
\hline 3 & $\mathrm{~N} 2-\mathrm{AM}=24$ & 12 & 500 & 168,5 & 452,38 & 1342,37 & $6 \mathrm{~cm}$ & $12 \mathrm{~cm}$ \\
\hline 3 & $\mathrm{~N} 3-\mathrm{AM}=36$ & 30 & 205 & 168,6 & 1130,97 & 1375,14 & $46 \mathrm{~cm}$ & $18 \mathrm{~cm}$ \\
\hline 4 & $4 \mathrm{~N} 1-\mathrm{AM}=12$ & 3 & 467 & 1600 & 113,09 & 33 & $6 \mathrm{~cm}$ & $6 \mathrm{~cm}$ \\
\hline 4 & N2 $2-A M=24$ & 12 & 450 & 1590 & 452,38 & 128,03 & $6 \mathrm{~cm}$ & $12 \mathrm{~cm}$ \\
\hline 4 & N3 $-A M=36$ & 30 & 135 & 1579 & 1130,97 & 96,69 & $6 \mathrm{~cm}$ & $18 \mathrm{~cm}$ \\
\hline
\end{tabular}

Tabela 1- Dados coletados (primeira parte)

Com isso, obtiveram-se valores de resistividade aparente para pontos descritos nas tabelas (1) e (2) para região de estudo. Para diversas profundidades tanto no sentido transversal, quando no sentido longitudinal, para as quatros linhas, distas $12 \mathrm{~cm}$ uma da outras, mostradas na Figura 1, para três profundidades diferentes, 6, 12 e 18 $\mathrm{cm}$.

\begin{tabular}{|c|c|c|c|c|c|c|c|c|}
\hline Linha & Sentido $\leftarrow$ & G & v & 1 & K & $\mathrm{Pa}$ & $x$ & p \\
\hline 1 & $\mathrm{~N} 1-\mathrm{AM}=12$ & 3 & 910 & 3,1 & 113,1 & 33631,33 & $6 \mathrm{~cm}$ & $6 \mathrm{~cm}$ \\
\hline 1 & $N 2-A M=24$ & 12 & 434 & 3 & 452,4 & 65883,53 & $6 \mathrm{~cm}$ & $12 \mathrm{~cm}$ \\
\hline & $\mathrm{N} 3-\mathrm{AM}=36$ & 30 & 140 & 2,9 & 1131 & 54224,58 & $6 \mathrm{~cm}$ & $18 \mathrm{~cm}$ \\
\hline 2 & $\mathrm{~N} 1-\mathrm{AM}=12$ & 3 & 708 & 37 & 113,1 & 2163,99 & $6 \mathrm{~cm}$ & $6 \mathrm{~cm}$ \\
\hline 2 & $N 2-A M=24$ & 12 & 365 & 42 & 452,4 & 3903,51 & $6 \mathrm{~cm}$ & $12 \mathrm{~cm}$ \\
\hline & $N 3-A M=36$ & 30 & 217 & 43 & 1131 & 5667,09 & $6 \mathrm{~cm}$ & $18 \mathrm{~cm}$ \\
\hline 3 & $\mathrm{~N} 1-\mathrm{AM}=12$ & 3 & 492 & 27 & 113,1 & 2060,75 & $6 \mathrm{~cm}$ & $6 \mathrm{~cm}$ \\
\hline 3 & $\mathrm{~N} 2-\mathrm{AM}=24$ & 12 & 325 & 26 & 452,4 & 5654,75 & $6 \mathrm{~cm}$ & $12 \mathrm{~cm}$ \\
\hline 3 & $\mathrm{~N} 3-\mathrm{AM}=36$ & 30 & 598 & 26 & 1131 & 26213,95 & $6 \mathrm{~cm}$ & $18 \mathrm{~cm}$ \\
\hline 4 & $\mathrm{~N} 1-\mathrm{AM}=12$ & 3 & 409 & 30 & 113,1 & 1541,79 & $6 \mathrm{~cm}$ & $6 \mathrm{~cm}$ \\
\hline 4 & $N 2-A M=24$ & 12 & 418 & 30 & 452,4 & 6388,33 & $6 \mathrm{~cm}$ & $12 \mathrm{~cm}$ \\
\hline 4 & $\mathrm{~N} 3-\mathrm{AM}=36$ & 30 & 1630 & 29 & 1131 & 5 & $6 \mathrm{~cm}$ & $18 \mathrm{~cm}$ \\
\hline
\end{tabular}

Tabela 2 - Dados coletados (segunda parte)

\section{Resultados}

O resumo dos resultados obtidos para resistividade aparente são exibidos na Tabela 3.

Tabela 3 - Resistividade aparente medida

\begin{tabular}{c|c|c|c|c|c} 
& $\mathrm{A} 1$ & $\mathrm{~A} 2$ & $\mathrm{~B} 1$ & $\mathrm{~B} 2$ & $\mathrm{C}$ \\
\hline Linha 1 & 2500.5 & 33631.33 & 20191.5 & 65833 & 79881.1 \\
\hline Linha 2 & 1102.7 & 2163.99 & 8131.4 & 3903.5 & 7704.3 \\
\hline Linha 3 & 2500.7 & 2060.75 & 1342.4 & 5654.7 & 13794.5 \\
\hline Linha 4 & 330.1 & 1541.79 & 128.03 & 6388.3 & 31722.8
\end{tabular}

Onde A1 = Profundidade 1 , Sentido $1 ; \mathrm{A} 2$ = Profundidade 1, Sentido 2; $\mathrm{B} 1=$ Profundidade 2 , Sentido $1 ; \mathrm{B} 2=$ Profundidade 2, Sentido 2; e C = Profundidade 3, Sentido único.

Para cada linha, um mapa foi elaborado. Para especulação dos valores nas demais áreas da caixa, utilizou-se o método de interpolação Krigagem, disponível no software Surfer. Com os valores interpolados, primeiramente criou-se um 'post map', através de comando com mesmo nome, para identificação dos pontos exatos os foram realizadas as medidas.

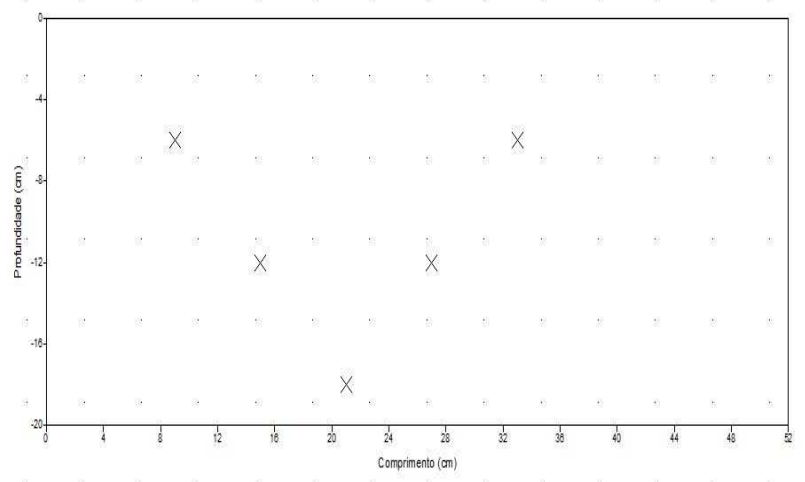

Figura 3 - Figura 2 - locais de medidas no modelo

Logo após, utilizou-se o comando 'contour map' para plotagem das curvas de contorno obtidas com a interpolação, identificando os locais de mesma resistividade aparente e classificando-os em escala de cores, sendo branco locais de menores resistividades e em vermelho pontos onde a resistividade aparente é mais elevada.

Apresenta-se nas figuras (4) a (7) as estimativas de resistividades aparente estimadas para as linhas de sondagem descritas na figura (2), percebe-se a tendência de aumento proporcional da resistividade com ao aumento da profundidade, com os pontos de maior resistividade centralizados nas camadas mais profundas do sistema. Esse comportamento era esperado devido a presença de materiais menos condutores, como as britas, no fundo da caixa. 


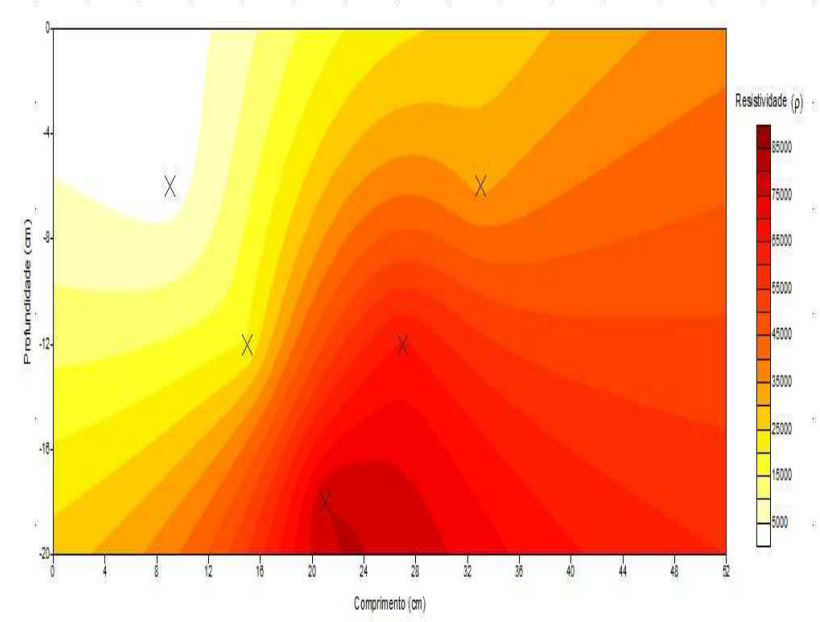

Figura 4 - Resistividade aparente estimada para a linha 1

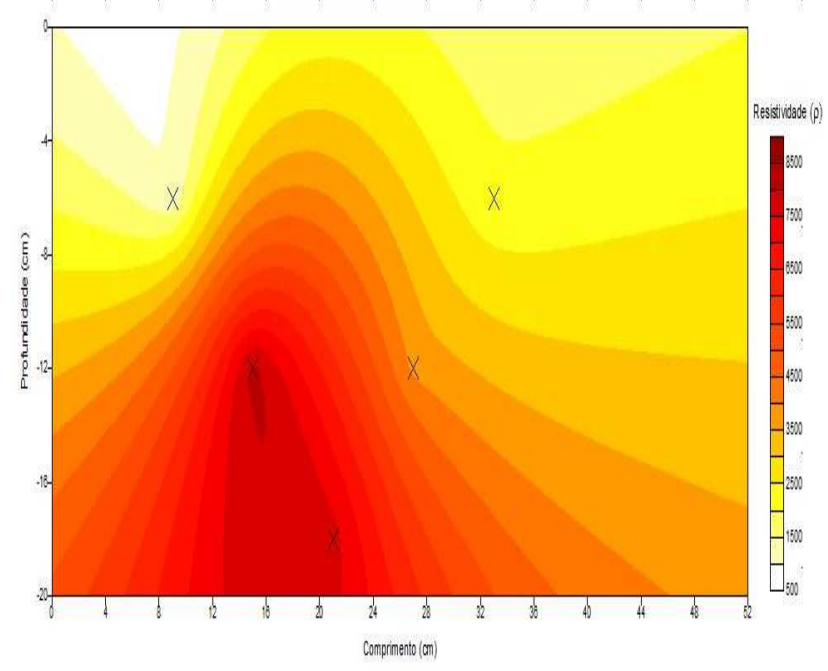

Figura 5 - Resistividade aparente estimada para a linha 2

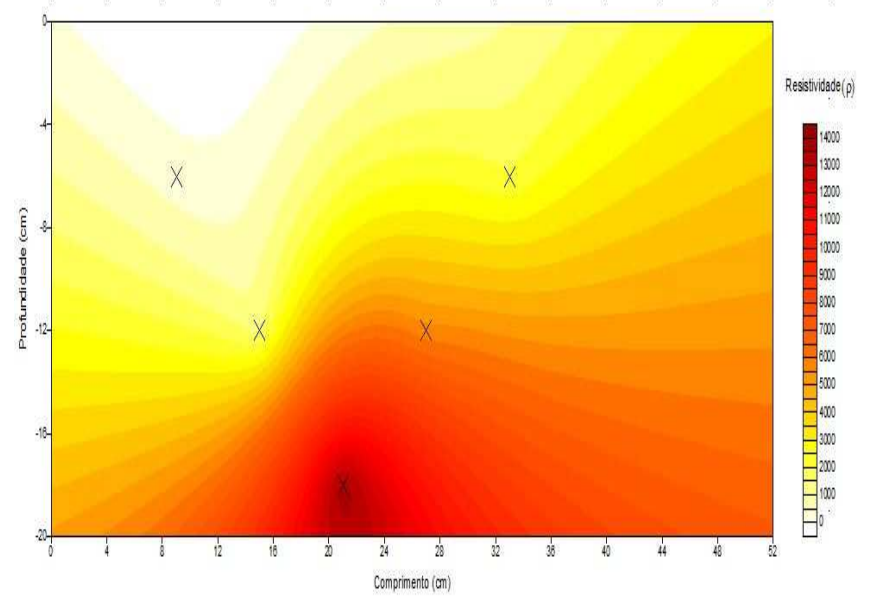

Figura 6 - Resistividade aparente estimada para a linha 3

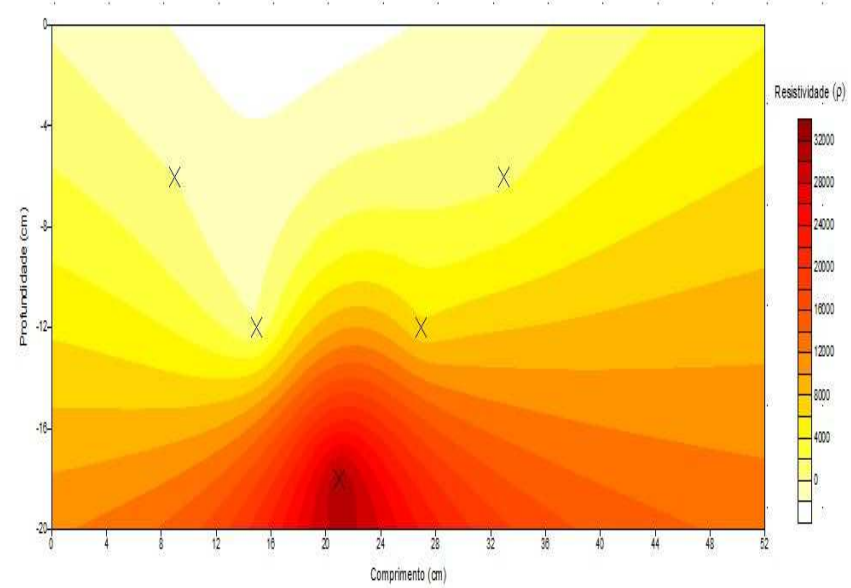

Figura 7 - Resistividade aparente estimada para a linha 4

\section{Discussão e Conclusões}

O sistema mostrou-se eficiente para a realização do método do caminhamento elétrico sem a necessidade de práticas de campo ou de utilização de equipamentos de alta precisão - e custo. Através de uma adaptação simples e eficaz, reduziu-se tempo e custo que seriam necessários para o ensinamento da mesma técnica em campo.

Os resultados obtidos para a resistividade apresentou variações elevadas entre as diferentes linhas. Isto pode ter sido consequência da pequena distância entre a fonte geradora de tensão e os medidores, em algumas das linhas. Além disto, a inexperiência dos estudantes na aplicação da técnica é um fator que contribui para a imprecisão dos dados, não sendo suficiente, no entanto, para a invalidação dos mesmos.

Na Figura 5, onde é apresentado o mapa obtido para a linha 2, observou-se o ponto de resistividade aparente máxima em posição diferente da apresentada nas outras linhas. Constatou-se então, que este ponto de maior resistividade era exatamente onde a pedra de maior dimensão havia sido inserida entre as camadas do solo. Isto comprova a eficácia do método, possibilitando identificação de áreas de menor condutância, característica de rochas não cristalinas e outros minérios.

Temos então que o sistema, guardadas as devidas proporções, é um substituto viável para ocasiões onde a prática de campo for inviável, por quaisquer motivos, apresentando resultados aceitáveis e permitindo que estudantes que não contam com os melhores equipamentos aprendam também de forma prática a realizar a técnica geofísica do Caminhamento Elétrico.

\section{Agradecimentos}

Aos demais discentes matriculados na disciplina, que contribuíram de forma essencial para a elaboração deste método alternativo de ensino e ao Técnico de Laboratório Fausto Cyrano, por sua disponibilidade e demais auxilio na para realização desse trabalho. 
Ao técnico Fausto Cyrano de Oliveira, pelo auxílio no manuseio dos equipamentos. A Deus, sobre todas as coisas.

\section{Referências}

BRAGA, Antônio Celso de Oliveira. 2007. Métodos Geoelétricos Aplicados. Universidade Estadual Paulista.

SBGf - Sociedade Brasileira de Geofíscia. Disponível em:

http://www.sbgf.org.br/geofisica/geofisica.html. Acesso em 18 abr. 2016.

UFPA - Universidade Federal do Pará. Faculdade de Geofísica. Disponível em: http://www.geofisica.ufpa.br/index.php/oquee. Acesso em 18 abr. 2016. 